\title{
IMAGE CHARACTERISTICS AND THEIR EFFECT ON DRIVING SIMULATOR VALIDITY
}

\author{
Hamish Jamson \\ Institute for Transport Studies, \\ University of Leeds, \\ Leeds, U.K. \\ Email: hamish@psyc.leeds.ac.uk
}

\begin{abstract}
Summary: Due to financial and computational limitations, the image quality presented in driving simulators is often a trade-off between resolution, pixel density and field of view. The current study examined this trade-off by investigating the effect of image resolution and horizontal field of view on the validity of the Leeds Driving Simulator. There were three levels of pixel density: low (3.6 arc min per pixel), high (2.6 arc min per pixel) and real world, and four levels of field of view: narrow $\left(50^{\circ}\right)$, medium $\left(120^{\circ}\right)$, wide $\left(230^{\circ}\right)$ and real world. Results seemed to show that widening the field of view improved the validity of speed choice and lane position between simulated and real world driving conditions, whilst there was no significant effect of image resolution.
\end{abstract}

\section{INTRODUCTION}

Most driving simulators use computer-generated images to display a virtual world to the driver. Due to financial, computational and rendering constraints, whilst maintaining a particular frame rate, the resolution and field of view of a system's image generator is finite. Normally, simulator designers must make a compromise and either manufacture a system with a wider field of view but low resolution, or one with a limited field of view but a higher resolution.

The average person can perceive an image that subtends an angle of less than one arc minute onto the foeval area of the retina (Kemeny, 1999); the pixel density of the image and its viewing distance defines this angle. Whilst state-of-the-art Head Mounted Displays (HMD) currently approach eye-limiting resolution, the majority of driving simulator applications avoid HMDs since it is generally considered unrealistic to expect normal individuals to drive naturally wearing such cumbersome and unfamiliar equipment. As a consequence, these simulators can only achieve a maximum of 3-4 arc minutes. Hence, the vast majority of simulators are incapable of providing its drivers with the same view that they would expect from experiences in the real world. Due to limitations in both projection and image generation hardware, it is far easier for simulator designers, by using three or more projected display channels to present an image, to produce fields of view exceeding the maximum human peripheral view of around $150^{\circ}$. Thus the majority of driving simulators have been developed with a wide field of view but low resolution.

In terms of simulator validity, Kappé et al. (1999) investigated the effect of horizontal field of view on subject performance. Subjects were required to perform a lane-keeping task whilst correcting for a slight side-wind. The results showed improved steering performance when the drivers experienced, at a constant resolution, a wide field of view as opposed to a narrow one. Staplin (1996) investigated the effect of display resolution on driver behaviour. Subject drivers were required to undergo the same gap acceptance task in the real world, and in a virtual 
environment of three varying degrees of image resolution. Results indicated the higher the resolution, the closer the drivers’ behaviour matched their real-life actions.

However, the important question to driving simulator designers is this: if only one is possible (high resolution, wide field of view), which is the more important in extracting realistic driver behaviour from a simulator? This is the question that this study attempts to answer by investigating the validity of the Leeds Driving Simulator with regard to the trade-off between horizontal field of view - subject drivers' “immersion” into the virtual environment - and the resolution of the display.

\section{THE LEEDS DRIVING SIMULATOR}

The Leeds Driving Simulator is currently based on a complete Rover 216GTi with all of its basic controls and dashboard instrumentation still fully operational. On a $2.5 \mathrm{~m}$ radius, cylindrical screen in front of the driver is projected a real-time, fully textured and anti-aliased, 3-D graphical scene of the virtual world. This scene is generated by a SGI Onyx ${ }^{2}$ Infinite Reality ${ }^{2}$ graphical workstation. The frame rate is a fixed to a constant $30 \mathrm{~Hz}$. A Roland digital sound sampler creates

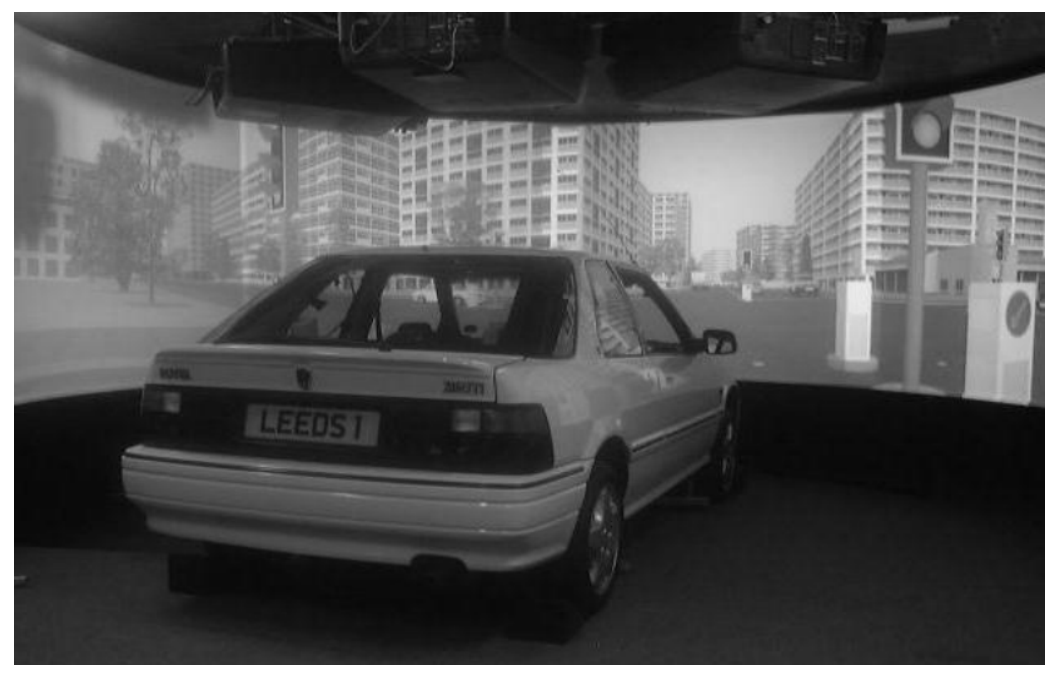

identity, position, speed). realistic sounds of engine and other noises via two speakers mounted close to each forward road wheel. Although the simulator is fixed-base, feedback is given by steering torques and speeds at the steering wheel. Data is collected at $30 \mathrm{~Hz}$ and includes information of the behaviour of the driver (i.e. driver controls), that of the car (position, speed, accelerations etc.) and other autonomous vehicles in the scene (e.g.

\section{Figure 1: The Leeds Driving Simulator}

\section{PREVIOUS VALIDATION STUDIES}

In the early days of development, the Leeds Driving Simulator simply had a single forward channel of $50^{\circ}$ horizontal field of view with a resolution of 1280x1024 pixels. A validation experiment was performed on this version of the simulator (Blana, 1999) by comparing simulator and real-life driving speed and lateral position on the same section of virtual and real road. The baseline data was collected using roadside video cameras, mounted at 21 different locations along a $6 \mathrm{~km}$ section of a single-carriageway, rural road (10 of these locations are used as the real-life driving behaviour baseline used in this study). Speed and lateral position was collected for 100 vehicles passing through each section in free-flow conditions. The same stretch of road layout was recreated in the simulator via data taken from 1:2500 Ordnance Survey maps. Digital 
images of roadside objects (trees, buildings, road-signs etc.), whose position had been surveyed from the real road, were used to create graphical objects within the simulation.

In this initial validation trial, the viewing distance of the projected image was $2.0 \mathrm{~m}$ and the image size was $1.98 \mathrm{~m}$ wide by $1.49 \mathrm{~m}$ high. This, with the image qualities mentioned above, equated to a pixel resolution of 2.7 arc min. 100 subjects (50 male and 50 female) took part. Reported results showed a good relative validity of speed, whilst in absolute terms, simulator drivers tended to drive curves slower and straights quicker than their real-world counterparts. This is a similar result to Harms’ (1996) validation of the VTI driving simulator. For vehicle lateral position, there was no absolute relationship between the two conditions, but in relative terms, drivers tended to follow the same path through the route.

Since this experiment was performed, the simulator has undergone two major upgrades. In the first of these, the simulator was configured with a wider field of view, made up of three channels, each with a resolution of 960x620 pixels (under half the resolution of the single channel condition). The three projected images were edge-blended to provide a near seamless total image of $120^{\circ}$. The viewing distance and image sizes were the same as in the single channel case, making the pixel resolution 3.6 arc min. The same validation test was performed on the simulator in this condition (Jamson, 2000) using 91 subjects (50 male and 41 female). Results suggested a general degradation in the validity of simulator drivers' lane position validity by the narrowing the apparent field of view.

\section{METHOD}

For the current study, a second simulator upgrade has allowed additional data to be collected. The projection system now consists of five forward channels, the front three of which at the original 1280x1024 pixels. Again the images are edge-blended, and along with two peripheral channels (640x480 each), the total horizontal field of view is now $230^{\circ}$. The design eye-point is at the centre of the $2.5 \mathrm{~m}$ cylindrical projection screen and each image size is $2.37 \mathrm{~m}$ wide by $1.78 \mathrm{~m}$ high, equating to a pixel resolution of $2.6 \mathrm{arc}$ min, assumed to be the same as the original version of the simulator (2.7 arc min). A rear view (640x480) is back projected onto a screen behind the car to provide an image seen through the vehicle's rear view and wing mirrors.

Along with data recorded during the previous two validation studies, a further 96 (50 male, 46 female) subjects performed the same simulated drive, in one of the remaining four conditions of image quality. A between-subjects comparison was made of the data relating to speed and lane position, for three levels of image resolution - low (3.6 arc min per pixel), high (2.6 arc min per pixel) and real world, and four levels of field of view - narrow $\left(50^{\circ}\right)$, medium $\left(120^{\circ}\right)$, wide $\left(230^{\circ}\right)$ and real world.

Two curves that made up an "S" shaped bend were selected for analysis. These curves were chosen since they gave the least satisfactory results in terms of absolute validity from the initial validation experiment. One straight section consisting of three data points was also selected for the same reason. Driver speed and lateral position on the real road were evaluated against the simulator conditions.

Data was recorded at four points associated with each curve: the approach, the entrance, the apex and the exit. The first curve (data points 1-4) was left-handed with a radius of $55.6 \mathrm{~m}$ and a length 
of $66.0 \mathrm{~m}$. The second curve (data points 5-7) was right-handed and followed immediately after the first curve; its radius and length were $108.2 \mathrm{~m}$ and $113.4 \mathrm{~m}$ respectively. The layout of the curves and the location of the data points can be seen in Figure 2. A further three data points (810 ) were located at $500 \mathrm{~m}$ intervals along a $1.5 \mathrm{~km}$ straight after the curved section.

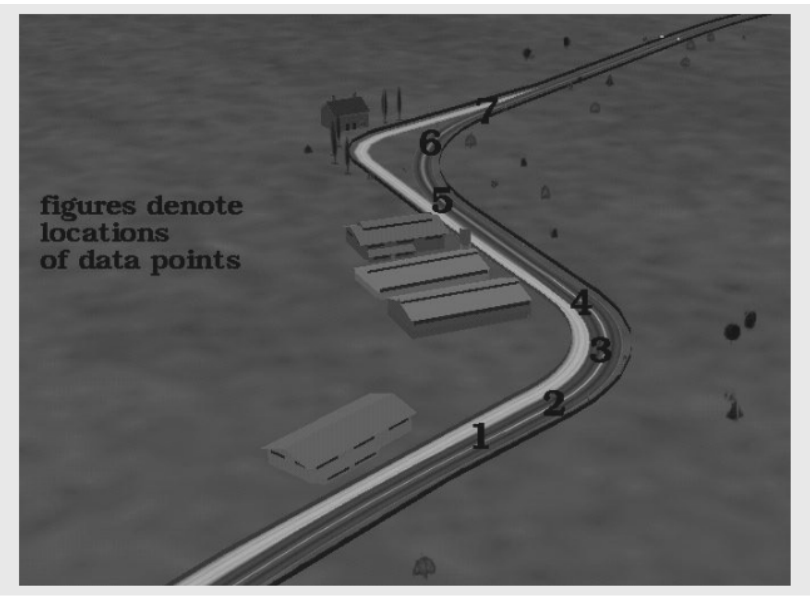

Figure 2: Layout of the curves and the location of the data points

\section{RESULTS}

A two-way ANOVA was performed to investigate the main effects of display resolution and horizontal field of view, followed by pairwise comparisons to highlight the simple effects. The assumptions of ANOVA were met. The dependant variables considered were spot speed and lateral position at the ten data points. They are considered separately here.

\section{Speed on curves}

\begin{tabular}{|c|c|c|c|c|c|c|c|}
\hline DATA & Real & \multicolumn{3}{|c|}{ High resolution } & \multicolumn{3}{c|}{ Low resolution } \\
\cline { 3 - 7 } POINT & world & $50^{\circ}$ & $120^{\circ}$ & $230^{\circ}$ & $50^{\circ}$ & $120^{\circ}$ & $230^{\circ}$ \\
\hline 1 (approach) & 64.4 & 53.2 & 51.5 & 57.2 & 59.4 & 47.0 & 54.9 \\
\hline 2 (entrance) & 50.3 & 46.4 & 45.6 & 52.0 & 49.2 & 43.3 & 47.4 \\
\hline 3 (apex) & 43.7 & 43.8 & 42.9 & 49.4 & 44.1 & 41.7 & 43.8 \\
\hline 4 (exit) & 45.7 & 45.3 & 45.3 & 51.5 & 46.9 & 43.6 & 51.5 \\
\hline 5 (entrance) & 50.3 & 43.9 & 45.1 & 50.2 & 47.4 & 42.3 & 46.5 \\
\hline 6 (apex) & 57.0 & 49.2 & 47.9 & 55.1 & 51.8 & 49.1 & 50.6 \\
\hline 7 (exit) & 56.3 & 56.0 & 55.3 & 61.6 & 58.3 & 55.7 & 57.3 \\
\hline
\end{tabular}

Table 1: mean speed (kph) at each data point (curves)

There was a main effect of field of view at points 1-6 $(\mathrm{p}<0.01)$, whilst there is no main effect of image resolution. Pairwise comparisons revealed that on the approach to the curves, there was a significant difference between real-life and simulated driving speeds in all simulated conditions, however this error was reduced in the $230^{\circ}$ condition. Similarly, on the entrance to the curves, widening the view to its maximum had the effect of reducing the significant differences between real-life and simulated driving speeds at narrower fields of view. The apexes and exits to the curves did not give consistent results. There were no simple effects of image resolution. 


\section{Speed on straight}

\begin{tabular}{|c|c|c|c|c|c|c|c|}
\hline \multirow{2}{*}{$\begin{array}{c}\text { DATA } \\
\text { POINT }\end{array}$} & Real & \multicolumn{3}{|c|}{ High resolution } & \multicolumn{3}{c|}{ Low resolution } \\
\cline { 3 - 8 } & world & $50^{\circ}$ & $120^{\circ}$ & $230^{\circ}$ & $50^{\circ}$ & $120^{\circ}$ & $230^{\circ}$ \\
\hline 8 & 80.7 & 93.9 & 92.5 & 95.0 & 89.9 & 91.1 & 90.2 \\
\hline 9 & 80.7 & 93.6 & 91.7 & 94.6 & 91.5 & 91.7 & 92.7 \\
\hline 10 & 83.4 & 94.2 & 91.1 & 94.7 & 94.1 & 92.7 & 93.2 \\
\hline
\end{tabular}

Table 2: mean speed (kph) at each data point (straight)

Drivers in all simulated conditions drove significantly faster than the real-life drivers $(\mathrm{p}<0.01)$. No speed differences could be attributed to simulator configuration.

\section{Lateral position on curves}

The lateral position values shown below in Table 3 were measured between the left-hand edge of the nearside, front road wheel and the edge of the paved area of the road (U.K. left side driving) .

\begin{tabular}{|c|c|c|c|c|c|c|c|}
\hline DATA & Real & \multicolumn{3}{|c|}{ High resolution } & \multicolumn{3}{c|}{ Low resolution } \\
\cline { 5 - 8 } POINT & world & $50^{\circ}$ & $120^{\circ}$ & $230^{\circ}$ & $50^{\circ}$ & $120^{\circ}$ & $230^{\circ}$ \\
\hline 1 (approach) & 737 & 1080 & 723 & 875 & 685 & 842 & 670 \\
\hline 2 (entrance) & 755 & 608 & 395 & 540 & 365 & 627 & 339 \\
\hline 3 (apex) & 271 & 310 & 245 & 320 & 208 & 387 & 218 \\
\hline 4 (exit) & 545 & 464 & 354 & 491 & 418 & 558 & 313 \\
\hline 5 (entrance) & 483 & 776 & 487 & 353 & 391 & 576 & 519 \\
\hline 6 (apex) & 822 & 1009 & 941 & 861 & 921 & 851 & 922 \\
\hline 7 (exit) & 713 & 1000 & 758 & 856 & 680 & 1018 & 752 \\
\hline
\end{tabular}

\section{Table 3: mean lateral position (mm) at each data point (curves)}

There was a main effect of image resolution on lateral position on the approach to the curve. However, since this only occurred at one of the data points, it is likely to be a spurious result. Pairwise comparisons revealed that on curve approach, curve entrance and final curve exit, drivers' lateral position was significantly different in the $50^{\circ}$ field of view condition. Widening the field of view progressively reduced the significance of this difference. There was no evidence of lateral position differences between simulated and real world conditions at either curve apex under any simulator configuration; neither were there any simple effects of resolution.

\section{Lateral position on straight}

\begin{tabular}{|c|c|c|c|c|c|c|c|}
\hline \multirow{2}{*}{$\begin{array}{c}\text { DATA } \\
\text { POINT }\end{array}$} & \multirow{2}{*}{$\begin{array}{c}\text { Real } \\
\text { world }\end{array}$} & \multicolumn{3}{|c|}{ High resolution } & \multicolumn{3}{c|}{ Low resolution } \\
\cline { 3 - 7 } & $50^{\circ}$ & $120^{\circ}$ & $230^{\circ}$ & $50^{\circ}$ & $120^{\circ}$ & $230^{\circ}$ \\
\hline 8 & 632 & 529 & 520 & 535 & 542 & 568 & 553 \\
\hline 9 & 723 & 575 & 590 & 642 & 579 & 581 & 590 \\
\hline 10 & 630 & 617 & 593 & 516 & 603 & 595 & 632 \\
\hline
\end{tabular}

Table 4: mean lateral position at each data point (straight) 
There was no main effect of either field of view or resolution on lane position on the straight section. Peculiarly, there was a difference between lane position in all simulator conditions and the real life condition only at data point 9 . This is most likely due to experimental error.

\section{CONCLUSIONS}

Ideally, a within-subjects design would have employed in order to reduce inherent betweensubject variability, however, this was impossible due to the longitudinal nature of this study. This may account for the somewhat unsystematic nature of the results. However, in general, it would appear that widening the field of view seems to improve the validity of speed choice and lane position between simulated and real world driving conditions.

There seems to be no corresponding improvement in validity from improving the image resolution, suggesting that a life-size projected image, even several magnitudes more coarse than reality, is sufficient at providing the necessary cues for lateral control of a driving simulator. However, poor image quality may affect driver performance in other situations not investigated in this study, e.g. overtaking decisions, interpretation of a distant road sign. Nevertheless, it is suggested that if a choice has to be made, driving simulator designers should compromise image resolution for widening their simulators' field of view.

\section{REFERENCES}

Blana, E. (1999). Behavioural Validation of a Fixed-base Driving Simulator. Paper presented at the Driving Simulation Conference 1999 (DSC 99). Paris, 7-8 ${ }^{\text {th }}$ July 1999.

Harms, L. (1996). Driving Performance on a Real Road and in a Driving Simulator. In Gale, A.G., Brown, I.D., Haslegrave, C.M., Moorhead, I. \& Taylor, S.P. (Eds.), Vision in Vehicles V. Elsevier Science B.V, Amsterdam.

Jamson, A.H. (2000). Driving Simulator Validity: Issues of Field of View and Resolution. Paper presented at the Driving Simulation Conference 2000 (DSC 2000). Paris, 4-6 ${ }^{\text {th }}$ September 2000.

Kappé, B., Korteling, J.E. and van Erp, J.B.F. (1999). Effects of Head-slaved and Peripherpal Images on Display Efficiency. Human Factors 41 (3), pp 453-466.

Kemeny, A. (1999). Simulation and Perception. Paper presented at the Driving Simulation Conference 1999 (DSC 99). Paris, 7-8 ${ }^{\text {th }}$ July 1999.

Staplin, L. (1996). Simulator and Field Measures of Driver Age Differences in Left-turn Gap Judgements. Transportation Research Record 1996 (1485), pp 49-55. 